\title{
22. \\ Ueber den inducirten Magnetismus eines unbegrenzten Cylinders von weichem Eisen.
}

(Von Herrn G. Kirchhoff, Professor an der Universität zu Breslau.)

Die Gleichungen, auf welche die von Poisson aufgestellte mathematische Theorie des in weichem Eisen inducirten Magnetismus geführt hat, sind für den Fall, dass der Eisenkörper ein Rotations-Ellipsoïd ist, von Neumann in (Crelle's Journal Bd. 37.) allgemein aufgelöset. Ein unbegrenzter Cylinder kann als ein Rotations-Ellipsoïd mit unendlich grosser Excentricität betrachtet werden; die Reihen-Entwickelungen von Neurnanı verlieren aber ihre Anwendbarkeit, wenn man in denselben die Excentricität unendlich gross setzt, ausser in dem Falle, dass der Mittelpunct der magnetisirenden Kräfte in unendlicher Entfernung liegt. Es soll hier die Lösung jener Gleichungen für einen unbegrenzten Cylinder unter der Annahme, dass die Mittelpuncte der inducirenden Kräfte im Endlichen liegen, auf einem andern Wege entwickelt werden.

\section{§. 1.}

Nach der Theorie von Poisson finden für jeden Punct eines Eisenkörpers, der durch Vertheilung magnetisirt ist, die beiden Gleichungen:

$$
\begin{gathered}
\text { () }=V+\varphi+U \\
U=-k \int \frac{d s}{\varepsilon} \cdot \frac{\partial \varphi}{d \boldsymbol{N} i}
\end{gathered}
$$

Statt. In demselben bedeutet $\boldsymbol{V}$ das Potential der magnetisirenden Kräfte, $k$ eine von der Natur des Eisens abhängige Constante, $\varphi$ eine Function welche den magnetischen Zustand des Körpers dadurch bestimmt, dass $k \frac{\partial \varphi}{\partial x}, k \frac{\partial \varphi}{\partial y}, k \frac{\partial \varphi}{\partial z}$ die magnetischen Momente, bezogen auf die Volumen-Einheit, in dem Puncte 
sind, welcher $x, y, z$ zu rechtwinkligen Coordinaten hat; $d s$ ist ein Element der Oberfläche des Eisenkörpers, $N_{i}$ ein unbestimmtes Stück der nach Innen gerichteten Normale von $d s, \frac{\partial \varphi}{\partial N_{i}}$ der Werth des nach $N_{i}$ genommenen Differentialquotienten von $\varphi$ für $N_{i}=0, \varepsilon$ die Entfernung des Elements $d s$ von dem Puncte, auf welchen sich $U$ bezieht; die Integration endlich ist über die ganze Oberfläche des Körpers auszudehnen. Hat man diesen beiden Gleichungen gemäss $\varphi$ bestimmt, so stellt der Ausdruck für $U$ in der Gleichung (2), sobald man in ihm unter $\varepsilon$ die Entfernung des Elements $d s$ von einem äusseren Puncte versteht, das Potential des magnetisirten Eisenkörpers in Beziehung auf diesen Punct dar.

Ich werde die Gleichungen (1 und 2) zunächst auf eine andere Form bringen, die ihre Lösung in dem gegenwärtigen Falle erleichtert.

Nach einem von Gauss bewiesenen Satze kann jedes Potential von Massen, die ausserhalb eines begrenzten Raumes liegen, bezogen auf einen Punct in diesem Raume, als herrührend von Massen auf der Oberfläche desselben betrachtet werden. $\quad V$ und $\varphi$ sind Potentiale von Massen, die ausserhalb des Eisenkörpers liegen; sie sollen als von Massenvertheilungen auf der Oberfläche des Körpers angesehen werden. Bezogen auf einen äusseren Punct sollen $\boldsymbol{V}$ und $\varphi$ die Potentiale von denselben Massenvertheilungen auf der Oherfläche bedeuten. Bezeichnet $N_{a}$ ein unbestimmtes Stück der nach Aussen gerichteten Normale von $d s$, und $\frac{\partial \varphi}{\partial N_{a}}$ den Werth des nach $N_{a}$ genommenen Differentialquotienten von $\varphi$ für $N_{a}=0$, so ist die Dichtigkeit der Masse, von welcher $\varphi$ herrührt, in dem Elemente $d s$ :

$$
-\frac{1}{4 \pi} \cdot\left(\frac{\partial \varphi}{d N_{i}}+\frac{\partial \varphi}{\partial N_{a}}\right)
$$

Die Dichtigkeit der Masse, von welcher $V$ herrührt, ist mit entsprechender Bezeichnung:

$$
-\frac{1}{4 \pi} \cdot\left(\frac{\partial V}{\partial N_{i}}+\frac{\partial V}{\partial N_{a}}\right)
$$

Ferner ist $\boldsymbol{U}$ ein Potential von Massen, die auf der Oberfläche liegen, und deren Dichtigkeit in dem Elemente $d s$,

$$
-k \frac{\partial \varphi}{\partial N_{i}}
$$

ist; die Dichtigkeit der.Massenvertheilung anf der Oberfläche, deren Potential $V+\varphi+U$ ist, ist daher:

Crelle's Journal f. d. M. Bd. XLVIII. Heft 4. 


$$
-\frac{1}{4 \pi} \cdot\left\{\frac{\partial V}{\partial N_{i}}+\frac{\partial V}{\partial N_{a}}+(1+4 \pi k) \frac{\partial \varphi}{\partial N_{i}}+\frac{\partial \varphi}{\partial N_{a}}\right\}
$$

Da das genannte Potential für alle Puncte des Eisenkörpers $=0$ ist, so muss diese Dichtigkeit für alle Puncte der Oberfläche verschwinden; d. h. es muss für alle diese Puncte die Gleichung:

$$
\text { (3.) } \frac{\partial V}{\partial N_{i}}+\frac{\partial V}{\partial N_{a}}+(1+4 \pi k) \frac{\partial \varphi}{\partial N_{i}}+\frac{\partial \varphi}{\partial N_{i}}+\frac{\partial \varphi}{\partial N_{a}}=0
$$

Statt finden. Diese Gleichung gilt zunächst nur für den Fall, dass der Eisenkörper ein endlicher ist; doch lässt sie sich auch auf einen unbegrenzten Cylinder anwenden, sobald die Kräfte, durch welche derselbe magnetisirt wird, von Polen ausgehen, die in der Endlichkeit liegen.

\section{§. 2.}

Um für den bezeichneten Fall aus der Gleichung (3) $\varphi$ ermitteln zu können, sollen zuerst Entwickelungen für ein Potential von Massen abgeleitet werden, die auf der Cylinderfläche liegen, dessen Werth in dieser Fläche gegeben ist; in Beziehung auf einen inneren und auf einen äusseren Punct.

Es seien $x, y, z$ die rechtwinkligen Coordinaten eines Puncts in Beziehung auf ein Coordinatensystem, dessen $\boldsymbol{x}$ Axe die Axe des Cylinders ist, und es sei $\boldsymbol{V}$ das Potential in Beziehung auf diesen Punct. Soll angedeutet werden, dass das Potential sich auf einen Punct innerhalb oder ausserhalb des Cylinders bezieht, so soll dem $V$ der Index $i$ oder $a$ beigefügt werden.

Es ist

$$
\frac{\partial^{2} V}{\partial x^{2}}+\frac{\partial^{2} V}{\partial y^{2}}+\frac{\partial^{2} V}{\partial z^{2}}=0
$$

oder, wenn man

$$
y=r \cos \vartheta, z=r \sin \vartheta
$$

setzt:

$$
\frac{\partial^{2} V}{\partial x^{2}}+\frac{\partial^{2} V}{\partial r^{2}}+\frac{1}{r} \cdot \frac{\partial V}{\partial r}+\frac{1}{r^{2}} \cdot \frac{\partial^{2} V}{\partial \vartheta^{2}}=0
$$

Es handelt sich darum $V_{i}$ so zu bestimmen, dass es innerhalb des Cylinders dieser partiellen Differentialgleichung genügt, und für die Oberfläche in eine gegebene Function von $x$ und $\vartheta$ übergeht; desgleichen $V_{a}$ so zu bestimmen, dass es 
ausserhalb des Cylinders derselben Gleichung Genüge thut, und für die Oberfläche derselben Function von $x$ und $\vartheta$ gleich wird.

Eine particuläre Lösung der Gleichung (4) ist folgende :

$$
V=W_{m} \cos m \vartheta+W_{m}^{\prime} \sin m \vartheta,
$$

wo $m$ eine ganze Zahl sein soll, $W_{m}$ und $W^{\prime}{ }_{m}$ Functionen von $x$ und $r$ bedeuten, die der Gleichung

genügen.

$$
\frac{\partial^{2} W_{m}}{\partial x^{2}}+\frac{\partial^{2} W_{m}}{\partial r^{2}}+\frac{1}{r} \cdot \frac{\partial W_{m}}{\partial r}-\frac{m^{2}}{r^{2}} \cdot W_{m}=0
$$

Man kann setzen :

$$
\begin{aligned}
& W_{m}=T_{n m}\left(G_{n m} \cdot \cos n x+H_{n m} \cdot \sin n x\right) \\
& W_{m}^{\prime}=T_{n m}\left(G_{n m}^{\prime} \cdot \cos n x+H_{n m}^{\prime} \cdot \sin n x\right),
\end{aligned}
$$

wo $n, G_{n m}, H_{n m}, G_{n m}^{\prime}, H_{n m}^{\prime}$ willkührliche Constanten sind und $\boldsymbol{T}_{n m}$ eine Function von $r$ ist, die der Gleichung:

$$
\frac{d^{2} T_{n m}}{d r^{2}}+\frac{1}{r} \cdot \frac{d T_{n m}}{d r}-\left(\frac{m^{2}}{r^{2}}+n^{2}\right) T_{n m}=0
$$

Genüge thut.

Ist $S_{m}(\varrho)$ eine Function von $\varrho$, welche die Gleichung:

$$
\varrho \frac{d^{2} S_{m}}{d \varrho^{2}}+(m+1) \frac{d S_{m}}{d \varrho}-S_{m}=0
$$

erfüllt, so genügt man der Gleichung (5) durch:

$$
T_{n m}=r^{m} \cdot S_{m}\left(\frac{n^{2} r^{2}}{4}\right)
$$

Das allgemeine Integral der Gleichung (6) ist von Kummer in diesem Journal, (Bd. 17, S. 229), in folgender Gestalt angegeben:

$$
S_{m}=a_{j} \psi\left(1+m_{\jmath} \rho\right)+b \varrho^{-m} \cdot \psi\left(1-m_{1} \varrho\right)
$$

hier sind $a$ und $b$ die willkührlichen Constanten, und die Function $\psi$ wird durch die Gleichung:

$$
\psi(\alpha, \varrho)=1+\frac{\varrho}{\alpha .1}+\frac{\varrho^{2}}{\alpha, \alpha+1.1 .2}+\frac{\varrho^{3}}{\alpha, \alpha+1 . \alpha+1.2 .3}+\text { etc. }
$$


definirt. Ich setze

dann ist

$$
\begin{gathered}
\psi(1+m, \varrho)=\boldsymbol{P}_{m}(\varrho) \\
\boldsymbol{T}_{n m}=r^{m} \boldsymbol{P}_{m}\left(\frac{n^{2} r^{2}}{4}\right)
\end{gathered}
$$

ein particuläres Integral der Gleichung (5), und zwar ein solches, welches für $\varrho=\infty$ unendlich gross wird, und für $\varrho=0$ endlich bleibt, oder verschwindet.

Kummer zeigt a. a. O., dass die Gleichung (6) auch durch:

$$
S_{m}=\int_{0}^{\infty} u^{-m-1} \cdot e^{-u} \cdot e^{-\frac{\varrho}{u}} d u,
$$

erfüllt wird, und er findet für den Werth dieses Integrals :

$$
\text { (7.) }=\Pi(-m-1) \psi(1+m, \varrho)+\Pi(m-1) \varrho^{-m} \psi(1-m, \varrho),
$$

wo $\Pi(z)$ die vón Gauss in seiner Abhandlung "circa seriem infinitam" .... in den Comment. Goetting. vom Jahre 1812, betrachtete Function bedeutet.

Der Ausdruck (7) soll durch

$$
\mathbf{Q}_{m}(\varrho)
$$

bezeichnet werden; dann ist auch

$$
T_{n m}=r^{m} Q_{m}\left(\frac{n^{2} r^{2}}{4}\right)
$$

ein particuläres Integral der Gleichung (5) und zwar ein solches, welches für $r=0$ unendlich gross wird, aber für $r=\infty$ verschwindet.

Der Ausdruck (7) stellt sich, wenn $m$ eine ganze Zahl ist, welcher Fall hier gerade in Betracht kommt, in einer unbestimmten Form dar. Man findet seinen Werth, indem man in ihm $m+\varepsilon$ statt $m$ schreibt, wo $\varepsilon$ eine unendlich kleine Grösse ist.

Setzt man, mit Gauss:

$$
\frac{d \log \Pi(z)}{d z}=\psi(z)
$$

und benutzt die Gleichungen :

$$
\Pi(z+1)=(z+1) \cdot \Pi(z), \Pi(0)=1 ;
$$

so ergiebt sich: 


$$
\Pi(-m-\varepsilon-1)=\frac{(-1)^{m+1}}{1.2 \ldots \ldots m} \cdot\left(\frac{1}{\varepsilon}-\psi(0)\right),
$$

und, wenn $m$ nicht $=0$ ist:

Dabei ist:

$$
ת(m+\varepsilon-1)=1.2 \ldots m-1[1+\varepsilon \cdot \psi(0)] .
$$

$$
\psi(0)=-0,5772157 \text {. }
$$

Ferner findet man:

$$
\psi(1+m+\varepsilon, \varrho)
$$

$=\psi(1+m, \varrho)-\varepsilon\left\{\varrho \frac{\frac{1}{m+1}}{m+1.1}+\rho^{2} \frac{\frac{1}{m+1}+\frac{1}{m+2}}{m+1 . m+2.1 .2}+\rho^{\frac{3}{3+1}+\frac{1}{m+1 . m+2 . m+3.1 .2 .3}+\cdots}+\frac{1}{m+3}\right\}$,

$\psi(1-m-\varepsilon, \varrho)=1-\frac{\varrho}{m-1.1}+\frac{\varrho^{2}}{m-1 . m-2.1 .2}-\ldots+\frac{(-1)^{m-1} \varrho^{m-1}}{m-1 . m-2 \ldots 2.1 .1 .2 \ldots m-2 . m-1}$

$$
\begin{aligned}
& +\frac{(-1)^{m} \varrho^{m}}{(1.2 \ldots m-1)^{2} m}\left\{\frac{1}{\varepsilon} \psi(1+m, \varrho)+\varrho \frac{1}{m+1.1}+\varrho^{2} \frac{1+\frac{1}{2}}{m+1 . m+2.1 .2}\right. \\
& \left.+\varrho^{3} \frac{1+\frac{1}{2}+\frac{1}{3}}{m+1 . m+2 . m+3.1 .2 .3}+\ldots . .\right\} .
\end{aligned}
$$

Endlich ist:

$$
\varrho^{-m-\varepsilon}=\varrho^{-m}(1-\varepsilon \log \varrho) .
$$

Substituirt man diese Werthe in den Ausdruck (7), so findet sich:

$$
\begin{aligned}
Q_{m}(\varrho)=1.2 \ldots m-1 . \varrho^{-m} & \left\{1-\frac{\varrho}{m-1.1}+\frac{\varrho^{2}}{m-1 . m-2.1 .2}-\ldots . .\right. \\
& \left.+\frac{(-1)^{m-1} \varrho^{m-1}}{m-1 . m-2 \ldots 2.1 .1 .2 \ldots m-2 . m-1}\right\}
\end{aligned}
$$

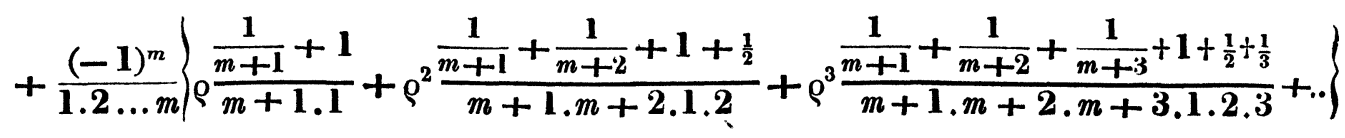

$+\frac{(-1)^{m}}{1.2 \ldots m}[2 \psi(\mathbf{0})-\log \varrho] \psi(1+m, \varrho)$.

Für den Fall $\boldsymbol{m}=\mathbf{0}$, hat man:

$$
\begin{aligned}
Q_{0}(\varrho) & =\Pi(-\varepsilon-1) \psi(1+\varepsilon, \varrho)+\Pi(\varepsilon-1) \rho^{-\varepsilon} \psi(1-\varepsilon, \varrho), \\
\Pi(-\varepsilon-1) & =-\frac{1}{\varepsilon}+\psi(0), \\
\Pi(\varepsilon-1) & =+\frac{1}{\varepsilon}+\psi(0),
\end{aligned}
$$




$$
\begin{aligned}
\psi(1+\varepsilon, \rho) & =\psi(1, \varrho)-\varepsilon\left\{\rho \frac{1}{1^{2}}+\rho^{2} \frac{1+\frac{1}{2}}{(1.2)^{2}}+\rho^{3} \frac{1+\frac{1}{2}+\frac{1}{3}}{(1.2 .3)^{2}}+\cdots\right\} \\
\psi(1-\varepsilon, \varrho) & =\psi(1, \varrho)+\varepsilon\left\{\rho \frac{1}{1^{2}}+\rho^{2} \frac{1+\frac{1}{2}}{(1.2)^{2}}+\rho^{3} \frac{1+\frac{1}{2}+\frac{1}{3}}{(1.2 .3)^{2}}+\ldots\right\} \\
\rho^{-\varepsilon} & =1-\varepsilon \log \varrho ;
\end{aligned}
$$

daraus folgt:

$$
Q_{0}(\varrho)=2\left\{\varrho \frac{1}{1^{2}}+\rho^{2} \frac{1+\frac{1}{2}}{(1.2)^{2}}+\varrho^{3} \frac{1+\frac{1}{2}+\frac{1}{3}}{(1.2 .3)^{2}}+\ldots\right\}+(2 \psi(0)-\log \varrho) \psi(1, \varrho) .
$$

Es giebt für $Q_{m}(\varrho)$ eine einfache semiconvergente Reihe, die nach fallenden Potenzen von $\varrho$ fortschreitet, und die hier beiläufig angeführt werden mag. Kummer hat in der angeführten Abhandlung gezeigt, dass

und dass

$$
Q_{m}(\varrho)=\frac{V \pi \rho^{-m} e^{-2} V}{2^{2 m-1} \Pi\left(-m-\frac{1}{2}\right)} \int_{0}^{\infty}\left(u+u^{2}\right)^{-m-\frac{1}{2}} e^{-4 u} \bigvee \rho d u,
$$

$$
\int_{0}^{\infty} \frac{u^{\alpha-1} e^{-u x} d u}{(1+u)^{\beta}}=\Pi(\alpha-1) x^{-\alpha}\left\{1-\frac{\alpha \cdot \beta}{1 \cdot x}+\frac{\alpha . \alpha+1 \cdot \beta \cdot \beta+1}{1.2 \cdot x^{2}}-\text { etc. }\right\}
$$

ist. Setzt man in der zweiten Gleichung:

$$
\alpha=\frac{1}{2}-m, \beta=\frac{1}{2}+m, x=\gamma_{\varrho},
$$

und verbindet sie mit der ersten, so erhält man :

$$
Q_{m}(\varrho)=V \pi . \varrho^{-\frac{2 m+1}{4}} \cdot e^{-2 \vee \rho}\left\{1-\frac{\left(1-4 m^{2}\right)}{1.16 V \varrho}+\frac{\left(1-4 m^{2}\right)\left(9-4 m^{2}\right)}{1.2 .(16 V \varrho)^{2}}-\frac{\left(1-4 m^{2}\right)\left(9-4 m^{2}\right)\left(25-4 m^{2}\right)}{1.2 .3 .(16 V \varrho)^{3}}+.\right\} .
$$

Es wurden die beiden folgenden particulären Integrale der Gleichung (4) gefunden:

$$
\begin{aligned}
V= & \cos m \vartheta r^{m} P_{m}\left(\frac{n^{2} r^{2}}{4}\right)\left(G_{n m} \cos n x+H_{n m} \sin n x\right) \\
& +\sin m \vartheta r^{m} P^{m}\left(\frac{n^{2} r^{2}}{4}\right)\left(G_{n m}^{\prime} \cos n x+H_{n m}^{\prime} \sin n x\right)
\end{aligned}
$$

und

$$
\begin{aligned}
V= & \cos m \vartheta r^{m} Q_{m}\left(\frac{n^{2} r^{2}}{4}\right)\left(I_{n m} \cos n x+K_{n m} \sin n x\right) \\
& +\sin m \vartheta r^{m} Q_{m}\left(\frac{n^{2} r^{2}}{4}\right)\left(I_{n m}^{\prime} \cos n x+K_{n m}^{\prime} \sin n x\right)
\end{aligned}
$$


wo die neu eingeführten Grössen $I_{n m}, K_{n m}, I_{n m}^{\prime}, K_{n m}^{\prime}$ willkührliche Constanten bezeichnen; das erste Integral wird $=\infty$ für $r=\infty$, und nicht $=\infty$ für $r=0$; das zweite wird $=\infty$ für $r=0$ und verschwindet für $r=\infty$. Von jeder dieser Lösungen kann in Beziehung auf $n$ das Integral, in Beziehung auf $m$ die Summe genommen werden; man kann setzen:

$$
\begin{aligned}
V_{i} & =\sum_{0}^{\infty} m \cos m \vartheta \int_{0}^{\infty} r^{m} P_{m}\left(\frac{n^{2} r^{2}}{4}\right)\left(G_{m}(n) \cos n x+H_{m}(n) \sin n x\right) d n \\
& +\sum_{1}^{\infty} m \sin m \vartheta \int_{0}^{\infty} r^{m} P_{m}\left(\frac{n^{2} r^{2}}{4}\right)\left(G_{m}^{\prime}(n) \cos n x+H_{m}^{\prime}(n) \sin n x\right) d n \\
V_{a} & =\sum_{0}^{\infty} m \cos m \vartheta \int_{0}^{\infty} r^{m} Q_{m}\left(\frac{n^{2} r^{2}}{4}\right)\left(I_{m}(n) \cos n x+K_{m}(n) \sin n x\right) d n \\
& +\sum_{1}^{\infty} m \sin m \vartheta \int_{0}^{\infty} r^{m} Q_{m}\left(\frac{n^{2} r^{2}}{4}\right)\left(I_{m}^{\prime}(n) \cos n x+K_{m}^{\prime}(n) \sin n x\right) d n,
\end{aligned}
$$

falls sich zeigen lässt, dass die Functionen von $n: G_{m}, H_{m}, G_{m}^{\prime}, H_{m}^{\prime}, I_{m}, K_{m}$, $I_{m}{ }_{m}, K^{\prime}{ }_{m}$ sich so bestimmen lassen, dass die Ausdrücke von $V_{i}$ und $V_{a}$ einer gegebenen Function von $x$ und $\vartheta$ gleich werden, wenn man in ihnen für $r$ den Radius des Cylinders setzt.

Es sei $\boldsymbol{R}$ der Radius des Cylinders, $\overline{\boldsymbol{V}}$ die Function von $x$ und $\boldsymbol{\vartheta}$, in welche $V_{i}$ und $V_{a}$ für $r=R$ übergehen sollen. Mit Hülfe des Fourier'schen Satzes lässt sich $\bar{V}$ auf folgende Form bringen:

$$
\left\{\begin{aligned}
\bar{V} & =\sum_{0}^{\infty} m \cos m \vartheta \int_{0}^{\infty}\left(A_{m}(n) \cos n x+B_{m}(n) \sin n x\right) d n \\
& +\sum_{1}^{\infty} m \sin m \vartheta \int_{0}^{\infty}\left(A_{m}^{\prime}(n) \cos n x+B_{m}^{\prime}(n) \sin n x\right) d n
\end{aligned}\right.
$$

Ist dieses geschehen, und nimmt man die Werthe von $G_{m}(n), H_{m}(n)$ u. s. w. aus den Gleichungen:

$$
\begin{aligned}
& \frac{G_{m}(n)}{A_{m}(n)}=\frac{H_{m}(n)}{B_{m}(n)}=\frac{G_{m}^{\prime}(n)}{A_{m}^{\prime}(n)}=\frac{H_{m}^{\prime}(n)}{B_{m}^{\prime}(n)}=\frac{1}{R^{m} P_{m}\left(\frac{n^{2} R^{2}}{4}\right)} \\
& \frac{I_{m}(n)}{A_{m}(n)}=\frac{K_{m}(n)}{B_{m}(n)}=\frac{I_{m}^{\prime}(n)}{A_{m}^{\prime}(n)}=\frac{K_{m}^{\prime}(n)}{B_{m}^{\prime}(n)}=\frac{1}{R^{m} Q_{m}\left(\frac{n^{2} R^{2}}{4}\right)},
\end{aligned}
$$

so wird der gestellten Forderung genügt. 
Hat man daher die Functionen $A_{m}(n), B_{m}(n), A_{m}^{\prime}(n), B_{m}^{\prime}(n)$ der Gleichung (8) gemäss bestimmt, so ist :

$$
\text { (9.) }\left\{\begin{aligned}
V_{i} & =\sum_{0}^{\infty} m \cos m \vartheta \int_{0}^{\infty} \frac{r^{m} P_{m}\left(\frac{n^{2} r^{2}}{4}\right)}{R^{m} P_{m}\left(\frac{n^{2} R^{2}}{4}\right)}\left(A_{m}(n) \cos n x+B_{m}(n) \sin n x\right) d n \\
& +\sum_{1}^{\infty} m \sin m \vartheta \int_{0}^{\infty} \frac{r^{m} P_{m}\left(\frac{n^{2} r^{2}}{4}\right)}{R^{m} \mathcal{L}_{m}\left(\frac{n^{2} R^{2}}{4}\right)}\left(A_{m}^{\prime}(n) \cos n x+B_{m}^{\prime}(n) \sin n x\right) d n, \\
V_{a} & =\sum_{0}^{\infty} m \cos m \vartheta \int_{0}^{\infty} \frac{r^{m} Q_{m}\left(\frac{n^{2} r^{2}}{4}\right)}{R^{m} Q_{m}\left(\frac{n^{2} R^{2}}{4}\right)}\left(A_{m}(n) \cos n x+B_{m}(n) \sin n x\right) d n \\
& +\sum_{1}^{\infty} m \sin m \vartheta \int_{0}^{\infty} \frac{r^{m} Q_{m}\left(\frac{n^{2} r^{2}}{4}\right)}{R^{m} Q_{m}\left(\frac{n^{2} R^{2}}{4}\right)}\left(A_{m}^{\prime}(n) \cos n x+B_{m}^{\prime}(n) \sin n x\right) d n .
\end{aligned}\right.
$$

\section{§. 3.}

Es sollen jetzt die gefundenen Entwickelungen benutzt werden, um aus der Gleichung (3) $\varphi$ zu bestimmen. Bei dieser Gleichung ist vorausgesetzt, dass sowohl $\boldsymbol{V}$ als $\varphi$ als Potentiale von Massen dargestellt sind, die auf der Oberfläche des Cylinders liegen. In Beziehung auf $\boldsymbol{V}$ wird diese Bedingung durch die in (9) aufgestellten Ausdrücke erfüllt; sie wird auch in Beziebung auf $\varphi$ erfüllt, wenn man, entsprechend

$$
\left\{\begin{aligned}
\varphi_{i} & =\sum_{0}^{\infty} m \cos m \vartheta \int_{0}^{\infty} \frac{r^{m} P_{m}\left(\frac{n^{2} r^{2}}{4}\right)}{R^{m} P_{m}\left(\frac{n^{2} R^{2}}{4}\right)}\left(C_{m}(n) \cos n x+D_{m}(n) \sin n x\right) d n \\
& +\sum_{1}^{\infty} m \sin m \vartheta \int_{0}^{\infty} \frac{r^{m} P_{m}\left(\frac{n^{2} r^{2}}{4}\right)}{R^{m} P_{m}\left(\frac{n^{2} R^{2}}{4}\right)}\left(C_{m}^{\prime}(n) \cos n x+D_{m}^{\prime}(n) \sin n x\right) d n \\
\varphi_{a} & =\sum_{0}^{\infty} m \cos m \vartheta \int_{0}^{\infty} \frac{r^{m} Q_{m}\left(\frac{n^{2} r^{2}}{4}\right)}{R^{m} Q_{m}\left(\frac{n^{2} R^{2}}{4}\right)}\left(C_{m}(n) \cos n x+D_{m}(n) \sin n x\right) d n \\
& +\sum_{1}^{\infty} m \sin m \vartheta \int_{0}^{\infty} \frac{r^{m} Q_{m}\left(\frac{n^{2} r^{2}}{4}\right)}{R^{m} Q_{m}\left(\frac{n^{2} R^{2}}{4}\right)}\left(C_{m}^{\prime}(n) \cos n x+D_{m}^{\prime}(n) \sin n x\right) d n
\end{aligned}\right.
$$


setzt, wo $C_{m}(n), D_{m}(n), C_{m}^{\prime}(n), D_{m}^{\prime}(n)$ Functionen bedeuten, die eben durch die Gleichung (3) ibre Bestimmung erhalten sollen.

Man hat:

$$
\begin{aligned}
& \frac{\partial V}{\partial N_{i}}=-\frac{\partial V_{i}}{\partial r}, \frac{\partial \varphi}{\partial N_{i}}=-\frac{\partial \varphi_{i}}{\partial r} \\
& \frac{\partial V}{\partial N_{a}}=\frac{\partial V_{a}}{\partial r}, \frac{\partial \varphi}{\partial N_{a}}=\frac{\partial \varphi_{a}}{\partial r},
\end{aligned}
$$

wenn man auf der rechten Seite dieser Gleichungen $r=R$ setzt; es ist daher:

$$
\begin{aligned}
\frac{\partial V}{\partial N_{i}}= & -\sum_{0}^{\infty} m \cos m \vartheta \int_{0}^{\infty} \frac{\partial \log \left(R^{m} P_{m}\left(\frac{n^{2} R^{2}}{4}\right)\right)}{\partial R}\left(A_{m}(n) \cos n x+B_{m}(n) \sin n x\right) d n \\
& -\sum_{1}^{\infty} m \sin m \vartheta \int_{0}^{\infty} \frac{\partial \log \left(R^{m} P_{m}\left(\frac{n^{2} R^{2}}{4}\right)\right)}{\partial R}\left(A_{m}^{\prime}(n) \cos x+B_{m}^{\prime}(n) \sin n x\right) d n
\end{aligned}
$$

und

$$
\begin{aligned}
\frac{\partial V}{\partial N_{a}}= & \sum_{0}^{\infty} m \cos m \vartheta \int_{0}^{\infty} \frac{\partial \log \left(R^{m} Q_{m}\left(\frac{n^{2} R^{2}}{4}\right)\right)}{\partial R}\left(A_{m}(n) \cos n x+B_{m}(n) \sin n x\right) d n \\
& +\sum_{1}^{\infty} m \sin m \vartheta \int_{0}^{\infty} \frac{\partial \log \left(R^{m} Q_{m}\left(\frac{n^{2} R^{2}}{4}\right)\right)}{\partial R}\left(A_{m}^{\prime}(n) \cos n x+B_{m}^{\prime}(n) \sin n x\right) d n .
\end{aligned}
$$

Aus diesen Ausdrücken erbält man die Ausdrücke für $\frac{\partial \varphi}{\partial N_{i}}$ und $\frac{\partial \varphi}{\partial N_{a}}$, wenn man statt der Buchstaben $A, B$ die Buchstaben $C, D$ setzt. Substituirt man diese Werthe in die Gleichung (3), so zeigt sich, dass dieselbe identisch erfüllt wird, wenn man:

$$
\begin{gathered}
\frac{C_{m}(n)}{A_{m}(n)}=\frac{D_{m}(n)}{B_{m}(n)}=\frac{C_{m}^{\prime}(n)}{A_{m}^{\prime}(n)}=\frac{D_{m}^{\prime}(n)}{B_{m}^{\prime}(n)} \\
=-\frac{\frac{\partial \log \left(R^{m} Q_{m}\left(\frac{n^{2} R^{2}}{4}\right)\right)}{\partial R}-\frac{\partial \log \left(R^{m} P_{m}\left(\frac{n^{2} R^{2}}{4}\right)\right)}{\partial \log \left(R^{m} Q_{m}\left(\frac{n^{2} R^{2}}{4}\right)\right)}}{\partial R}-(1+4 \pi k) \frac{\partial \log \left(R^{m} P_{m}\left(\frac{n^{2} R^{2}}{4}\right)\right)}{\partial R}
\end{gathered}
$$

naacht. Führt man in diesen Ausdruck

$$
\varrho=\frac{n^{2} R^{2}}{4}
$$

Crelle's Journal f. d. M. Bd. XLVIII. Heft 4. 
ein, so wird derselbe

$$
=-\frac{1}{1-4 \pi k \frac{Q_{m}(\varrho)\left(m P_{m}(\varrho)+2 \varrho \frac{d P_{m}(\varrho)}{d \varrho}\right)}{2 \varrho\left(P_{m}(\varrho) \frac{d Q_{m}(\varrho)}{d \varrho}-Q_{m}(\varrho) \frac{d P_{m}(\varrho)}{d \varrho}\right)}} .
$$

Nun genügen $\boldsymbol{P}_{m}(\varrho)$ und $Q_{m}(\varrho)$ der Differentialgleichung (6); hieraus ergiebt sich leicht:

$$
\boldsymbol{P}_{m}(\varrho) \frac{d Q_{m}(\varrho)}{d \varrho}-Q_{m}(\varrho) \frac{d P_{m}(\varrho)}{d \varrho}=\frac{c}{\varrho^{m+1}}
$$

wo $c$ eine von $\varrho$ unabhängige Grösse bedeutet. Man findet den Werth derselben, wenn man $\varrho$ als unendlich klein voraussetzt. Unter dieser Annahme ist, nach den im vorigen Paragraph über $\boldsymbol{P}_{m}(\varrho)$ und $\boldsymbol{Q}_{m}(\varrho)$ gemachten Angaben :

$$
\begin{aligned}
& \boldsymbol{P}_{m}(\varrho)=1 \quad, \frac{d P_{m}(\varrho)}{d \varrho}=\frac{1}{m+1}, \\
& Q_{m}(\varrho)=\frac{1.2 \ldots m-1}{\varrho^{m}}, \frac{d Q_{m}(\varrho)}{d \varrho}=-\frac{1.2 \ldots m}{\varrho^{m+1}} .
\end{aligned}
$$

Für $\boldsymbol{m}=\mathbf{0}$ ist aber:

$$
Q_{0}(\varrho)=-\log \varrho \quad, \quad \frac{d Q_{0}(\varrho)}{d \varrho}=-\frac{1}{\varrho}
$$

hieraus ergiebt sich:

also

$$
\begin{aligned}
& \boldsymbol{P}_{m}(\varrho) \frac{d Q_{m}(\varrho)}{d \varrho}-Q_{m}(\varrho) \frac{d P_{m}(\varrho)}{d \varrho}=-\frac{1.2 \ldots m}{\varrho^{m+1}} \\
& \boldsymbol{P}_{0}(\varrho) \frac{d Q_{0}(\varrho)}{d \varrho}-Q_{0}(\varrho) \frac{d P_{0}(\varrho)}{d \varrho}=-\frac{1}{\varrho},
\end{aligned}
$$

$$
\left\{\begin{array}{l}
\frac{C_{m}(n)}{A_{m}(n)}=\frac{D_{m}(n)}{B_{m}(n)}=\frac{C_{m}^{\prime}(n)}{A_{m}^{\prime}(n)}=\frac{D_{m}^{\prime}(n)}{B_{m}^{\prime}(n)} \\
=-\frac{1}{1+2 \pi k \frac{\varrho^{m} Q_{m}(\varrho)\left(m P_{m}(\varrho)+2 \varrho \frac{d P_{m}(\varrho)}{d \varrho}\right)}{1.2 \ldots m}} \\
\text { und } \\
\frac{C_{0}(n)}{A_{0}(n)}=\frac{D_{0}(n)}{B_{0}(n)}=\frac{C^{\prime}{ }_{0}(n)}{A_{0}^{\prime}(n)}=\frac{D_{0}^{\prime}(n)}{\bar{B}_{0}^{\prime}(n)} \\
=-\frac{1}{1+4 \pi k \varrho Q_{0}(\varrho) \frac{d P_{0}(\varrho)}{d \rho}} .
\end{array}\right.
$$


Durch die Gleichungen $(12,11)$ und die erste der Gleichungen $(10)$ ist die Function $\varphi$ für alle Puncte im Innern des Eisenkörpers, und dadurch der magnetische Zustand desselben bestimmt.

Es ist von Interesse, auch das Potential des magnetisirten Eisenkörpers in Beẓjehung auf einen äusseren Punct, d. h. die Grösse $U_{a}$, zu ermitteln. Wenn $V$ und $\varphi$ als Potentiale von Massen dargestellt sind, die auf der Oberfläche des Körpers liegen (wie es im Früheren geschehen ist), so findet die Gleichung:

$$
V_{a}+\varphi_{a}+U_{a}=\mathbf{0}
$$

Statt. Daraus ergiebt sich leicht:

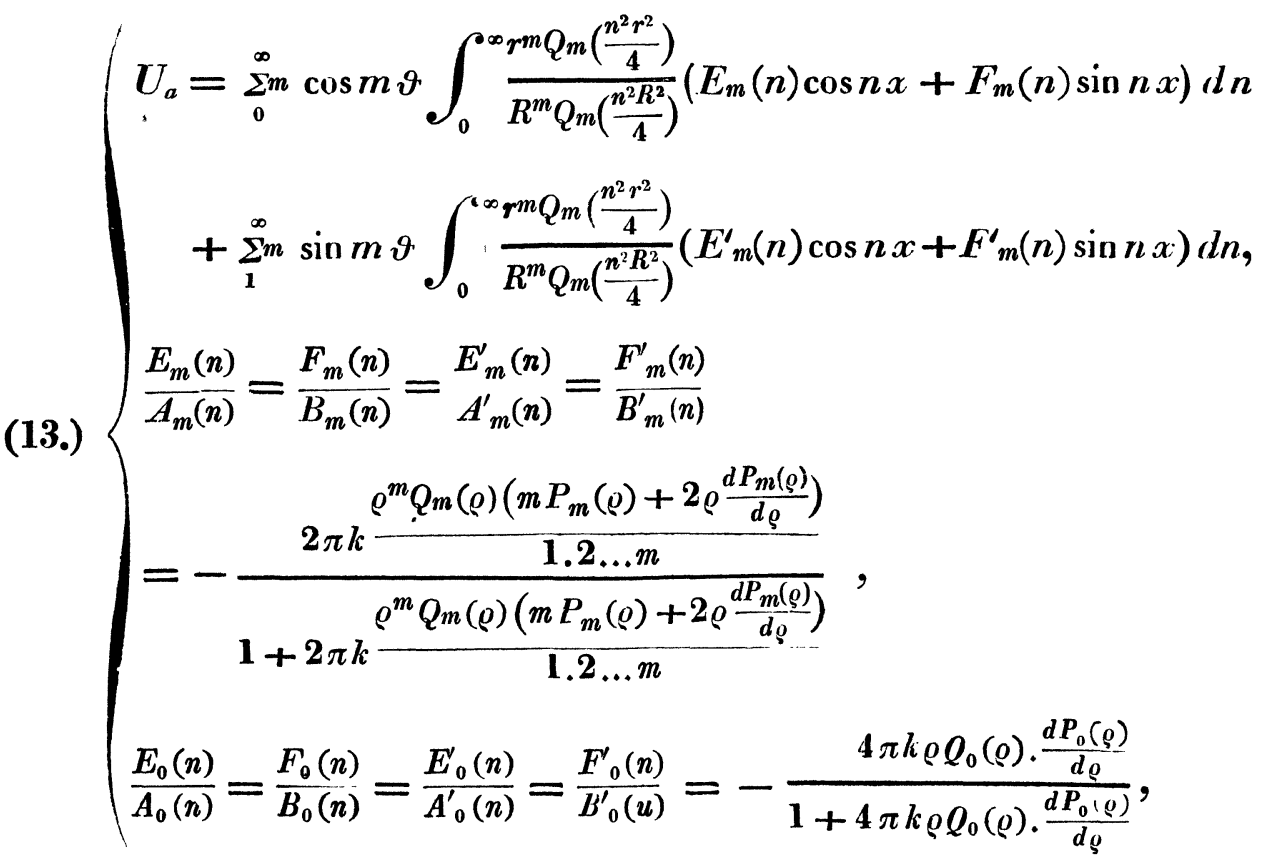

wo $\varrho$ wiederum den in der Gleichung (11) angegebenen Werth hat.

§. 4.

Die aufgestellten Formeln sind nicht gültig, wenn die Kräfte, welche den Eisencylinder magnetisiren, ganz, oder zum Theil von einem elektrischen Strome herrühren, der den Cylinder umkreiset. In diesem Falle haben die Integrale, welche nach dem Fourier'schen Satze den mit $A, B, A^{\prime}, B^{\prime}$ bezeichneten Grössen 
gleich sind, keine angebbaren Werthe, weil das Potential $V$ nicht für $x=-\infty$ und $x=+\infty$ verschwindet. Das Potential eines geschlossenen electrischen Stromes, dessen Intensität nach electro-magnetischem Maasse $=1$ ist, ist gleich der Fläche, welche der Kegel, der zur Spitze den Punct hat, auf den das Potential sich bezieht, und der durch die Stromcurve geht, von der Kugelfläche ausschneidet, die um denselben Punct mit dem Radius 1 beschrieben ist. Man kann nach Willkür den einen oder den andern der beiden Theile, in welche die Kugel durch den Kegel zerlegt wird, als das Potential ansehen; nur muss man beachten, dass das Potential sich stetig ändert, wenn der Punct auf welchen es sich bezieht, seine Lage stetig ändert. (Vergl. Neumann's Allgemeine Gesetze der inducirten electrischen Ströme; Abh. der Berl. Ak. 1845. p. 73). Es folgt daraus, dass, wenn ein Strom von der Intensität 1, der den Cylinder einmal umfliesst, beim Magnetisiren mitwirkt, das Potential $V^{\prime}$ so angenommen werden kann, dass es für $x=-\infty$ verschwindet, dass es dann aber für $x=+\infty$ den Werth $4 \pi$ erhält.

Es soll jetzt gezeigt werden, wie in einem solchen Falle der magnetische Zustand des Cylinders und seine Wirkung nach Aussen sich ermitteln lässt.

Es möge das System von Körpern, von welchen die magnetisirenden Kräfte ausgehen, als verschiebbar in der Pichtung der $x$ Axe betrachtet werden, und es sei $\xi$ die $x$ Ordinate eines mit diesem Systeme fest verbundenen Puncts. Alsdann werden $V, \varphi$ und $\boldsymbol{U}$ Functionen, nicht bloss von $x, r$ und $\vartheta$, sondern auch von $\xi$ sein. Differentïrt man die Gleichungen (1 und 2) nach $\xi$, so erhält man Gleichungen, die sich von diesen nur dadurch unterscheiden, dass $V, \varphi$ und $U$ durch $\frac{\partial V}{\partial \xi}, \frac{\partial \varphi}{\partial \xi}$ und $\frac{\partial U}{\partial \xi}$ ersetzt sind. Daraus folgt, dass, wenn $\frac{\partial V}{\partial \xi}$ das Potential der magnetisirenden Kräfte ist, $\frac{\partial \varphi_{i}}{\partial \xi}$ diejenige Function ist, deren Differentialquotienten die magnetischen Momente im Innern des Cylinders bestimmen, und $\frac{\partial U_{a}}{\partial \xi}$ das Potential des magnetisirten Cylinders in Beziehung auf einen äusseren Punct. Es verschwindet aber $\frac{\partial V}{\partial \xi}$ sowohl für $x=-\infty$ als für $x=+\infty$, auch wenn die Kräfte, deren Potential $\boldsymbol{V}$ ist, von electrischen Strömen herrühren; daher können die im vorigen Paragraphen entwickelten Formeln benutzt werden, um $\frac{\partial \varphi_{i}}{\partial \xi}$ und $\frac{d U_{a}}{d \xi}$ aus $\frac{\partial V}{\partial \xi}$ zu finden. Bestimmt man die Functionen $A, B, A^{\prime}, B^{\prime}$ so, dass die Gleichung erfüllt wird, in welche (8) übergeht, wenn man $\bar{V} \operatorname{durch} \frac{\partial \bar{V}}{\partial \xi}$ ersetzt, so sind die in $\left(10\right.$ u. 13) für $\varphi_{i}$ und $U_{a}$ aufgestellten Ausdrücke $=\frac{\partial \varphi_{i}}{\partial \xi}$ und $=\frac{\partial U_{a}}{\partial \xi}$. 
Die Grössen $V, \varphi, U$ sind aber Functionen von $x-\xi$; est ist daher:

$$
\frac{\partial V}{\partial \xi}=-\frac{\partial V}{\partial x}, \frac{\partial \varphi}{\partial \xi}=-\frac{\partial V}{\partial x}, \frac{\partial U}{\partial \xi}=-\frac{\partial U}{\partial x} .
$$

Ersetzt man also in (8) $\bar{V}$ durch $\frac{\partial \bar{V}}{\partial x}$, so geben die in (10 und 13) für $\varphi_{i}$ und $U_{a}$ aufgestellten Ausdrücke die Werthe von $\frac{\partial \varphi_{i}}{\partial x}$ und $\frac{\partial U_{a}}{\partial x}$. Aus diesen erhält man durch Integration $\varphi_{i}$ und $U_{a}$ selbst. Die hierbei eintretenden Constanten der Integration können Functionen von $r$ und $\vartheta$ sein. Um den magnetischen Zustand des Cylinders und seine Wirkung nach Aussen zu ermitteln, ist es nur nöthig die Differentialquotienten derselben nach $r$ und $\vartheta$ zu kennen; diese werden dadurch bestimmt, dass $\frac{\partial \varphi_{i}}{\partial r}, \frac{\partial \varphi_{i}}{\partial \vartheta}, \frac{\partial U_{a}}{\partial r}, \frac{\partial U_{a}}{\partial \vartheta}$ für $x= \pm \infty$ verschwinden müssen.

\section{§. 5.}

Es soll der Fall näher untersucht werden, wo die magnetisirenden Kräfte von einem Kreisstrome herrühren, dessen Mittelpunct in der Axe des Cylinders liegt, und dessen Ebene senkrecht auf dieser Axe steht. Der Strom habe die Intensität 1 und den Radius $s$; der Mittelpunct desselben sei der Anfangspunct der Coordinaten; dann ist für $r=0$ :

also

$$
V=2 \pi\left(1+\frac{x}{V\left(s^{2}+x^{2}\right)}\right)
$$

$$
\frac{\partial V}{\partial x}=\frac{2 \pi s^{2}}{\left(s^{2}+x^{2}\right)^{\frac{3}{2}}} \text {. }
$$

Bedeutet $f(x)$ eine Function, für welche $f(-x)=f(+x)$ ist, so hat man nach dem Fourier'schen Satze :

$$
f(x)=\frac{2}{\pi} \int_{0}^{\infty} d n \cdot \cos n x \int_{0}^{\infty} d \alpha \cdot \cos n \alpha \cdot f(\alpha)
$$

die Function $\frac{\partial V}{d x}$ genügt der für $f(x)$ ausgesprochenen Bedingung, daher ist für $r=0$ :

$$
\frac{\partial V}{\partial x}=4 \int_{0}^{\infty} d n \cdot \cos n x \int_{0}^{\infty} \frac{s^{2} d \alpha \cdot \cos n \alpha}{\left(s^{2}+\alpha^{2}\right)^{\frac{3}{2}}}
$$


oder, wenn man $s \alpha$ vor $\alpha$ schreibt:

$$
\frac{d V}{d x}=4 \int_{0}^{\infty} d n \cdot \cos n x \int_{0}^{\infty} \frac{d \alpha \cdot \cos n s \alpha}{\left(1+\alpha^{2}\right)^{\frac{3}{2}}} .
$$

Das nach $\alpha$ zu nehmende Integral kann man auf das Integral:

$$
\int_{0}^{\infty} \frac{\cos p \alpha \cdot d \alpha}{V\left(1+\alpha^{2}\right)}
$$

zurückführen, und dieses lässt sich auf folgende Weise finden:

Es ist:

$$
\int_{0}^{\infty} e^{-a^{2} y^{2}} d y=\frac{1}{2} V_{\pi} \cdot \frac{1}{a}
$$

also, wenn $a^{2}=1+a^{2}$ gemacht wird:

$$
\frac{1}{V\left(1+a^{2}\right)}=\frac{2}{V \pi} \int_{0}^{\infty} e^{-y^{2}} \cdot e^{-a^{2} y^{2}} d y,
$$

mithin:

$$
\begin{aligned}
\int_{0}^{\infty} \frac{\cos p \alpha \cdot d \alpha}{V\left(1+\alpha^{2}\right)} & =\frac{2}{V \pi} \cdot \int_{0}^{\infty} e^{-y^{2}} d y \int_{0}^{\infty} \cos p \alpha \cdot e^{-a^{2} y^{2}} d a \\
& =\int_{0}^{\infty} e^{-y^{2}} d y \cdot \frac{1}{y} \cdot e^{-\frac{p^{2}}{4 y^{2}}}
\end{aligned}
$$

oder, wenn man $y^{2}=u$ setzt:

$$
=\frac{1}{2} \int_{0}^{\infty} \frac{1}{u} e^{-u} \cdot e^{-\frac{p^{2}}{4 u}} d u,
$$

oder endlich bei der in (§. 2.) gebrauchten Bezeichnung:

$$
\int_{0}^{\infty} \frac{\cos p \alpha \cdot d \alpha}{V\left(1+\alpha^{2}\right)}=\frac{1}{2} Q_{0}\left(\frac{p^{2}}{4}\right) \text {. }
$$

Statt dieser Gleichung kann man auch

$$
\int_{0}^{\infty} \frac{\cos \alpha d \alpha}{V\left(p^{2}+\alpha^{2}\right)}=\frac{1}{2} Q_{0}\left(\frac{p_{2}}{4}\right)
$$

schreiben. Differentiirt man nun nach $p$, so erhält man : 
22. Kirchhoff, zur Theorie des Magnetismus.

oder, wenn man

$$
-\int_{0}^{\infty} \frac{p \cos \alpha \cdot d \alpha}{\left(p^{2}+\alpha^{2}\right)^{\frac{3}{2}}}=\frac{1}{2} \frac{d}{d p} \cdot Q_{0}\left(\frac{p^{2}}{4}\right),
$$

$$
\frac{d Q_{0}(\varrho)}{d \varrho}=Q_{0}^{\prime}(\varrho)
$$

setzt:

$$
\int_{0}^{\infty} \frac{\cos p \alpha \cdot d \alpha}{\left(1+\alpha^{2}\right)^{\frac{3}{2}}}=-\frac{p^{2}}{4} Q_{0}^{\prime}\left(\frac{p^{2}}{4}\right)
$$

Der Gleichung (14) zufolge ist daher für $r=0$ :

$$
\frac{\partial V}{d x}=-4 \int_{0}^{\infty} d n \cos n x \cdot \frac{n^{2} s^{2}}{4} Q_{0}^{\prime}\left(\frac{n^{2} s^{2}}{4}\right) \text {. }
$$

Für ein unbestimmtes $r$ hat man aber nach der ersten der Gleichungen (9):

$$
\frac{\partial V}{\partial x}=\int_{0}^{\infty} d n \cos n x \frac{P_{0}\left(\frac{n^{2} r^{2}}{4}\right)}{P_{0}\left(\frac{n^{2} R^{2}}{4}\right)} A_{0}(n) ;
$$

die Vergleichung dieser beiden Ausdrücke zeigt, dass:

$$
A_{0}(n)=-4 P_{0}\left(\frac{n^{2} R^{2}}{4}\right) \cdot \frac{n^{2} s^{2}}{4} Q_{0}^{\prime}\left(\frac{n^{2} s^{2}}{4}\right)
$$

Setzt man, entsprechend der Gleichung (15):

$$
\frac{d P_{0}(\varrho)}{d \varrho}=P_{0}^{\prime}(\varrho)
$$

so erhält man aus den Gleichungen (10, 11, 12 und 13):

$$
\begin{aligned}
\frac{\partial \varphi_{i}}{\partial x} & =4 \int_{0}^{\infty} \frac{\left.d n \cdot \cos n x P_{0}\left(\frac{n^{2} r^{2}}{4}\right) \cdot \frac{n^{2} s^{2}}{4}\right) Q_{0}^{\prime}\left(\frac{n^{2} s^{2}}{4}\right)}{1+4 \pi k\left(\frac{n^{2} R^{2}}{4}\right) P_{0}^{\prime}\left(\frac{n^{2} R^{2}}{4}\right) Q_{0}\left(\frac{n^{2} R^{2}}{4}\right)} \\
\text { (16.) } \frac{\partial U_{a}}{\partial x} & =16 \pi k \cdot \int_{0}^{\infty} \frac{d n \cdot \cos n x \cdot Q_{0}\left(\frac{n^{2} r^{2}}{4}\right) \cdot P_{0}\left(\frac{n^{2} R^{2}}{4}\right) \cdot \frac{n^{2} R^{2}}{4} \cdot P_{0}^{\prime}\left(\frac{n^{2} R^{2}}{4}\right) \cdot \frac{n^{2} s^{2}}{4} \cdot Q_{0}\left(\frac{n^{2} s^{2}}{4}\right)}{1+4 \pi k \frac{n^{2} R^{2}}{4} \cdot P_{0}^{\prime}\left(\frac{n^{2} R^{2}}{4}\right) \cdot Q_{0}\left(\frac{n^{2} R^{2}}{4}\right)} .
\end{aligned}
$$

Ich füge noch folgende Bemerkung hinzu.

Aus dem für $A_{0}(n)$ gefundenen Werthe folgt, dass $-\frac{1}{4} \frac{\partial V_{i}}{\partial x}$ dembestimmten Integrale

$$
\int_{0}^{\infty} d n \cos n x \cdot P_{0}\left(\frac{n^{2} r^{2}}{4}\right) \cdot \frac{n^{2} s^{2}}{4} Q_{0}\left(\frac{n^{2} s^{2}}{4}\right)
$$


gleich ist; dieselbe Grösse lässt sich aber auch durch elliptische Integrale ausdrükken. Thut man dies, so erhält man den Werth dieses bestimmten Integrals durch elliptische Integrale ausgedrückt. Betrachtet man statt des Potentials eines Kreisstromes das Potential einer Kreislinie, die auf der anziehenden Masse gleichmässig vertheilt ist, so gelangt man auf ähnliche Weise zu der Gleichung

$$
\int_{0}^{\infty} d n \cos n x P_{0}\left(\frac{n^{2} r^{2}}{4}\right) \cdot Q_{0}\left(\frac{n^{2} s^{2}}{4}\right)=\frac{2}{V\left[x^{2}+(s+r)^{2}\right]} \int_{0}^{\frac{\pi}{2}} \frac{d \varphi}{\sqrt{\left(1-\frac{4 s r}{x^{2}+(s+r)} \sin ^{2} \varphi\right)}} .
$$

Bei der Ableitung dieser Gleichung ist vorausgesetzt, dass $s>r$ ist; sie gilt auch nur unter dieser Voraussetzung, da das Integral auf ihrer linken Seite keinen angebbaren Werth mehr hat, wenn $s<r$ ist. Es geht dies aus der folgenden Betrachtung hervor.

Für ein unendlich grosses $\varrho$ ist:

$$
\begin{aligned}
& Q_{0}(\varrho)=V \pi \frac{1}{\sqrt{4} \varrho} e^{-2 V \varrho} \\
& P_{0}(\varrho)=\frac{1}{2 \sqrt{ } \pi} \cdot \frac{1}{\sqrt[V]{ } \varrho} e^{2 V \varrho},
\end{aligned}
$$

und daher für ein unendlich grosses $n$ :

$$
P_{0}=\left(\frac{n^{2} r^{2}}{4}\right) \cdot Q_{0}\left(\frac{n^{2} s^{2}}{4}\right)=\frac{1}{V(r s)} \cdot \frac{1}{n} e^{-n(s-r)}
$$

dieser Ausdruck verschwindet, wenn $s>r$, er wird aber unendlich gross, wenn $s<r$ ist.

\section{§. 6.}

Liegen die Mittelpuncte der magnetisirenden Kräfte in unendlicher Entfernung, so sind die hier angegebenen Entwickelungen nicht anwendbar. Aber in diesem Falle lässt sich der magnetische Zustand des Cylinders finden, indem man ihn als ein Rotations. Ellipsoïd mit unendlich grosser Excentricität betrachtet.

Ein Ellipsoïd wird durch Kräfte, die für alle seine Puncte gleich sind, gleichmässig magnetisirt, d. h. so, dass die magnetischen Momente, bezogen auf die Volumen-Einheit, constant sind. Bedeuten $\alpha, \beta, \gamma$ diese magnetischen Momente, sind ferner die Haupt-Axen des Ellipsoïds die Coordinaten-Axen und 
$a, b, c$ die Componenten der magnetisirenden Kraft, so ist, wie Neumann gezeigt hat:

$$
\left\{\begin{array}{l}
\alpha=\frac{k a}{1+k A^{0}}, \\
\beta=\frac{k b}{1+k B^{0}}, \\
\gamma=\frac{k c}{1+k C^{0}} ;
\end{array}\right.
$$

wo $A^{0}, B^{0}, C^{0}$ gewisse, von den Verhältnissen der Haupt-Axen abbängige, Grössen bezeichnen. Für ein Rotations-Ellipsoïd hat Neumann die Werthe dieser Grössen aufgestellt; führt man in diese Werthe die Bedingung ein, dass das Ellipsoïd ein unbegrenzter Cylinder sei, dessen Axe die $x$ Axe ist, so erhält man:

$$
A^{0}=\mathbf{0} \quad, \quad B^{0}=C^{0}=2 \pi ;
$$

für den Cylinder ist daher:

$$
\begin{aligned}
& a=k a \\
& \beta=\frac{k b}{1+2 \pi k} \\
& \gamma=\frac{k b}{1+2 \pi k} .
\end{aligned}
$$

Kennt man den magnetischen Zustand, in welchen eine Eisenmasse durch Kräfte versetzt wird, deren Mittelpunct in unendlicher Entfernung sich befindet, so ist man im Stande, die magnetischen Momente derselben Eisenmasse anzugeben für den Fall, dass sie durch irgend welche Kräfte magnetisirt ist. Man gelangt hierzu durch Anwendung folgenden Satzes:

Das Potential einer Eisenmasse, die magnetisirt ist durch ein System I, in Beziehung auf ein System II, ist gleich dem Potential derselben Eisenmasse, wenn sie magnetisirt ist durch das System II, in Beziehung auf das System I.

Dieser Satz lässt sich wie folgt beweisen.

Es sollen $V_{1}, \varphi_{1}, U_{1}$, die drei Grössen $V, \varphi, U$ für den Fall bezeichnen, dass die Eisenmasse durch das System I magnetisirt ist, $V_{2}, \varphi_{2}, U_{2}$ dieselben Grössen für den Fall, dass das System II den Magnetismus her.vorruft. Das Potential der durch $I$. magnetisirten Eisenmasse in Beziehung auf einen äusseren Punct ist dann:

Crelle's Journal f. d. M. Bd. XLVIII. Heft 4.

$$
U=-k \int \frac{d s}{\varepsilon} \cdot \frac{\partial \varphi}{\partial N_{i}}
$$


wo $r$ die Entfernung des in Rede stehenden Puncts von dem Elemente der Oberfläche, $d s$, bedeutet; das Potential der Eisenmasse in Beziehung auf das System II, welches durch $U_{1,2}$ bezeichnet werden mag, ist daher:

$$
\boldsymbol{U}_{1,2}=-k \int d s . \nu_{2} \cdot \frac{\partial \varphi_{1}}{\partial N_{i}},
$$

wo $V_{2}$ auf den Ort des Elements $d s$ zu beziehen ist.

Für alle Puncte der Eisenmasse, also auch für ihre Oberfläche ist aber:

daraus folgt:

$$
V_{2}+\varphi_{2}+U_{2}=0
$$

$$
U_{1,2}=k \int d s \cdot \varphi_{2} \cdot \frac{\partial \varphi_{1}}{\partial N_{i}}+k \int d s . U_{2} \cdot \frac{\partial \varphi_{1}}{\partial N_{i}} \text {. }
$$

Substituirt man in diese Gleichung:

$$
U_{2}=-k \int \frac{d s^{\prime}}{\varepsilon} \cdot \frac{\partial \varphi_{2}}{\partial N_{i}}
$$

wo $d s^{\prime}$ ein zweites Element der Oberfläche, $N_{i}^{\prime}$ die Normale desselben und $\varepsilon$ die Entfernung der beiden Elemente $d s$ und $d s^{\prime}$ bedeutet, so erhält man:

$$
\boldsymbol{U}_{1,2}=k \int d s, \varphi_{2} \cdot \frac{\partial \varphi_{1}}{\partial N_{i}}-k^{2} \iint \frac{d s d s^{\prime}}{\varepsilon} \cdot \frac{\partial \varphi_{1}}{\partial N_{i}} \cdot \frac{\partial \varphi_{2}}{\partial N_{i}} ;
$$

der Ausdruck rechts in dieser Gleichung ändert seinen Werth nicht, wenn man die Indices 1 und 2 vertauscht, denn es ist

$$
\int d s \varphi_{2} \frac{\partial \varphi_{1}}{\partial N_{i}}=\int d s \varphi_{i} \frac{\partial \varphi_{2}}{\partial N_{i}}
$$

weil $\varphi_{1}$ und $\varphi_{2}$ Potentiale von Massen ausserhalb des Eisenkörpers sind; hieraus geht die Richtigkeit des ausgesprochenen Satzes hervor.

Dieser Satz soll nun auf den Fall angewendet werden, dass das System II ein Magnetpol ist, der unendlich entfernt liegt. In diesem Falle ist

$$
V_{2}=-(a x+b y+c z)
$$

zu setzen, wo $a, b$, $c$ die Componenten der Kraft bedeuten, die der Pol ausübt; es wird also:

$$
\begin{aligned}
U_{1,2} & =k \int d s(a x+b y+c z) \frac{\partial \varphi_{1}}{\partial N_{i}} \\
& =a k \int d s \cdot x \cdot \frac{\partial \varphi_{1}}{\partial N_{i}}+b k \int d s \cdot y \cdot \frac{\partial \varphi_{1}}{\partial N_{i}}+c k \int d s . z \cdot \frac{\partial \varphi_{1}}{\partial N_{i}}
\end{aligned}
$$


Die Coefficienten von $a, b, c$ rechts in dieser Gleichung sind die negativ genommenen magnetischen Momente des Eisenkörpers, wenn er durch die Kräfte, deren Potential $V_{1}$ ist, magnetisirt ist; bezeichnet man diese Momente durch $L_{1}$, $M_{1}, N_{1}$, und beachtet, dass sie unabhängig von $a, b, c$ sind, so erhält man:

$$
\begin{gathered}
\boldsymbol{L}_{1}=-\frac{\partial U_{1,2}}{\partial a} \\
M_{1}=-\frac{\partial U_{1,2}}{\partial b} \\
N_{1}=-\frac{\partial U_{1,2}}{\partial c} .
\end{gathered}
$$

Nach dem vorausgeschickten Satze ist aber:

$$
U_{1,2}=-k \int d s . V_{1} \cdot \frac{\partial \varphi_{2}}{\partial N_{i}}
$$

oder auch, wenn man durch do ein Element des Volumens des Eisenkörpers bezeichnet:

$$
\dot{U}_{1,2}=k \int d v\left(\frac{\partial V_{1}}{\partial x} \cdot \frac{\partial \varphi_{2}}{\partial x}+\frac{\partial V_{1}}{\partial y} \cdot \frac{\partial \varphi_{2}}{\partial y}+\frac{\partial V_{1}}{\partial z} \cdot \frac{\partial \varphi_{2}}{\partial z}\right)
$$

Substituirt man einen dieser beiden Werthe von $U_{1,2}$ in die Gleichungen für $\boldsymbol{L}_{1}$, $M_{1}, N_{1}$, so erhält man diese durch $\varphi_{1}$ ausgedrückt; welches als bekannt vorausgesetzt ist. Bei der Ausführung der Differentiationen nach $a, b, c$ verschwinden diese Grössen aus der Rechnung, da $V_{1}$ unabhängig und $\varphi_{2}$ eine lineare Function von ihnen ist.

Für ein Ellipsoïd, dessen Haupt - Axen die Coordinaten-Axen sind, ist:

$$
\varphi_{2}=\frac{a x}{1+k A_{0}}+\frac{b y}{1+k B_{0}}+\frac{c z}{1+k C_{0}}
$$

daraus ergiebt sich - wenn der Index 1 jetzt weggelassen wird:

$$
\begin{aligned}
L & =-\frac{k}{1+k A_{0}} \int d v \frac{\partial V}{\partial x}, \\
M & =-\frac{k}{1+k B_{0}} \int d v \frac{\partial V}{\partial y}, \\
N & =-\frac{k}{1+k C_{0}} \int d v \frac{\partial V}{\partial z} .
\end{aligned}
$$

Diese Ausdrücke sind identisch mit denen, welche Neumann auf eine andere Weise hergeleitet hat. 
Für den unbegrenzten Cylinder werden die Ausdrücke für die magnetischen Momente folgende:

$$
\left\{\begin{array}{l}
L=-k \int d v \frac{d V}{\partial x}, \\
M=-\frac{k}{1+2 \pi k} \int d v \frac{d V}{d y}, \\
N=-\frac{k}{1+2 \pi k} \int d v \frac{d V}{d z} .
\end{array}\right.
$$

Die erste dieser Gleichungen lässt sich folgendermassen schreiben:

$$
\boldsymbol{L}=-k \int d y \cdot d z\left(V_{\infty}-V_{-\infty}\right),
$$

wenn man durch $V_{\infty}$ und $V_{-\infty}$ die Werthe von $V$ für $x=\infty$ und $x=-\infty$ bezeichnet. Rührt $V$ nur von Magnetpolen her, die im Endlichen liegen, so ist $V_{\infty}=V_{-\infty}=0$, also auch $L=0$; ist der Cylinder durch electrische Ströme, die ihn umkreisen, magnetisirt, so ist, welches auch die Gestalt der Stromcurven sei:

$$
V_{\infty}-V_{-\infty}=4 \pi \cdot I_{\eta}
$$

wo $I$ die Intensität der Ströme und $\eta$ die Zahl der Windungen bedeutet, in welchen dieselben um den Cylinder geführt sind. Es wird dann also:

$$
L=-4 \pi^{2} k \cdot R^{2} I_{\eta}
$$

wo $\boldsymbol{R}$ wieder den Radius des Cylinders bezeichnet.

\section{§. 7.}

Der durch die Gleichungen $\left(10,11\right.$ und 12) bestimmte Ausdruck von $\varphi_{i}$ vereinfacht sich sehr, wenn man den Radius des Cylinders, $\boldsymbol{R}$, unendlich klein setzt. In diesem Falle wird $\varrho$, d. h. $\frac{n^{2} R^{2}}{4}$, unendlich klein; die Gleichungen(12) geben dann für jedes $m$, ausser für $m=0$ :

und

$$
\frac{C_{m}(n)}{A_{m}(n)}=\frac{D_{m}(n)}{B_{m}(n)}=\frac{C_{m}^{\prime}(n)}{A_{m}^{\prime}(n)}=\frac{D_{m}^{\prime}(n)}{B_{m}^{\prime}(n)}=-\frac{1}{1+2 \pi k}
$$

$$
\frac{C_{0}(n)}{A_{0}(n)}=\frac{D_{0}(n)}{B_{0}(n)}=\frac{C_{0}^{\prime}(n)}{A_{0}^{\prime}(n)}=\frac{D_{0}^{\prime}(n)}{B_{0}^{\prime}(n)}=-1 .
$$


Bezeichnet man das dem $m=0$ entsprechende Glied der in (9) gegebenen Entwickelung von $V_{i}$ mit $V_{i}^{(0)}$, d. h. setzt man

$$
\frac{1}{2 \pi} \int_{0}^{2 \pi} V_{i} d \vartheta=V_{i}^{(0)}
$$

so giebt der in (10) aufgestellte Ausdruck für $\varphi_{i}$ :

$$
\text { (19.) } \quad \varphi_{i}=-V_{i}^{(0)}-\frac{V_{i}-V_{i}^{(0)}}{1+2 \pi k} .
$$

Diese Gleichung ist zunächst nur für den Fall bewiesen, in welchem die Gleichungen (9 und 10) gelten, d. h. für den Fall, dass der Cylinder durch Magnetpole, die in der Endlichkeit liegen, magnetisirt ist. Aber auch wenn ein elektrischer Strom, der den Cylinder umfliesst, bei seiner Magnetisirung mitwirkt, kann man sich der Gleichung (19) bedienen, um seinen Zustand kennen zu lernen. In diesem Falle ist nämlich :

$$
\frac{\partial \varphi_{i}}{\partial x}=-\frac{\partial V_{i}^{(0)}}{\partial x}-\frac{1}{1+2 \pi k}\left(\frac{\partial V_{i}}{\partial x}-\frac{\partial V_{i}^{(0)}}{\partial x}\right) \text {. }
$$

Düch Integration nach $x$ erhält man hieraus:

$$
\varphi_{i}=C-V_{i}^{(0)}-\frac{\nabla_{i}-V_{i}^{(0)}}{1+2 \pi k}
$$

die Grösse $C$ muss hier von $r$ und $\vartheta$ unabhängig sein, da $\frac{\partial V_{i}^{(0)}}{\partial r}, \frac{\partial V_{i}}{\partial r}$ und $\frac{\partial V_{i}}{\partial \vartheta}$ für $x= \pm \infty$ verschwinden, und für dieselben Werthe von $x$ auch $\frac{\partial \psi_{i}}{\partial r}$ und $\frac{\partial \varphi_{i}}{\partial \vartheta}$ verschwinden sollen; daher wenn die Grösse $C$ auch nicht $=0$ ist, hat ihr Werth doch keinen Einfluss auf die Differentialquotienten von $\varphi_{i}$, die allein den magnetischen Zustand bestimmen.

Mit Hülfe der Gleichung (19) lassen sich leicht die magnetischen Momente des ganzen Cylinders berechnen; man gelangt dann für den hier betrachteten Fall zu den halben Ausdrücken, die in (18) angegeben sind.

Rühren die magnetisirenden Kräfte von einem kreisförmigen elektrischen Strome her, wie er in ( $\$$. 5) betrachtet worden ist, so wird die Wirkung des $\mathrm{Cy}$ linders nach Aussen durch die Gleichung (16) bestimmt. Durch die Annahme, dass $\boldsymbol{R}$ unendlich klein ist, geht diese Gleichung in

$$
\frac{\partial U_{a}}{\partial x}=4 \pi k R^{2} \int_{0}^{\infty} d n \cdot \cos n x \cdot n^{2} Q_{0}\left(\frac{n^{2} r^{2}}{4}\right) \frac{n^{2} s^{2}}{4} Q_{0}^{\prime}\left(\frac{n^{2} s^{2}}{4}\right)
$$


über. Hieraus lässt sich leicht der Ausdruck für das Potential des magnetisirten Cylinders in Beziehung auf einen zweiten Kreisstrom bilden, dessen Mittelpunct in der Axe des Cylinders liegt, und dessen Ebene senkrecht auf dieser Axe steht. E's sei $\boldsymbol{W}$ dieses Potential, der Radius des zweiten Kreisstromes sei $=r$, seine Intensität $=1$, und $x$ der Abstand der Mittelpuncte der beiden Ströme; dann hat man, mit Rücksicht darauf, dass die Summe der Massen, deren Potential $\boldsymbol{U}_{a}$ ist, verschwindet:

$$
W=2 \pi \int_{r}^{\infty} \frac{\partial U_{a}}{\partial x} r d r .
$$

Da $Q_{0}(\varrho)$ der Differentialgleichung genügt, die man aus (6) erhält, wenn man $m=0$ setzt, so ist :

und daher

$$
Q_{0}(\rho)=\frac{d}{d \varrho} \rho Q_{0}^{\prime}(\varrho),
$$

also

$$
\frac{n^{2} r}{2} Q_{0}\left(\frac{n^{2} r^{2}}{4}\right)=\frac{d}{d r} \cdot \frac{n^{2} r^{2}}{4} Q_{0}^{\prime}\left(\frac{n^{2} r^{2}}{4}\right)
$$

Hieraus folgt:

$$
\int_{r}^{\infty} r d r n^{2} Q_{0}\left(\frac{n^{2} r^{2}}{4}\right)=-2 \frac{n^{2} r^{2}}{4} Q_{0}^{\prime}\left(\frac{n^{2} r^{2}}{4}\right) \text {. }
$$

$$
W=-16 \pi^{2} k R^{2} \int_{0}^{\infty} d n \cos n x \frac{n^{2} r^{2}}{4} Q_{0}^{\prime}\left(\frac{n^{2} r^{2}}{4}\right) \frac{n^{2} s^{2}}{4} Q_{0}^{\prime}\left(\frac{n^{2} s^{2}}{4}\right)
$$

Anhang.

Die Grundgleichungen der Poisson'schen Theorie des inducirten Magnetismus, nämlich die Gleichungen ( 1 und 2), können aus der Annahme hergeleitet werden, dass das magnetische Moment einer Kugel von weichem Eisen, bezogen auf die Volumen-Einheit, wenn die Kugel unter dem Einfluss einer für alle ihre Puncte constanten Kraft sich befindet, gleich der Intensität dieser Kraft, multiplicirt mit einer constanten Grösse ist. · Die Versuche von Müller und VVeber haben aber gezeigt, dass diese Annahme nicht zulässig ist, wenn die magnetisirende Kraft sehr stark ist, und haben zu dem Schlusse geführt, dass die Grösse, die nach der Poisson'schen Theorie constant sein soll, von der Intensität der magnetisirenden Kraft abhängt, in' der Weise, dass sie um so kleiner wird, je mehr die Kraft wächst. Ich will die Gleichungen ableiten, die bei dieser Annahme an die Stelle,der Gleichungen (1 und 2) gesetzt werden müssen.

Es seien $\alpha, \beta, \gamma$ die magnetischen Momente, reducirt auf die Einheit des Volumens, einer Kugel, auf die eine constante magnetisirende Kraft wirkt, deren 
Componenten $X, Y, Z$ sind. Wenn die Coëncitivkraft des Eisens $=\mathbf{0}$ ist, d. h. wenn der magnetische Zustand desselben allein von den Kräften abhangt, die in dem betrachteten Augenblicke auf dasselbe wirken, so muss die magnetische Axe der Kugel mit der Richtung der Kraft zusammenfallen; es muss daher

$$
\alpha: \beta: \gamma=X: Y: Z
$$

sein, und folglich nach der gemachten Annahme:

$$
\left\{\begin{array}{l}
\alpha=X f\left(V X^{2}+Y^{2}+Z^{2}\right) \\
\beta=Y f\left(V X^{2}+Y^{2}+Z^{2}\right) \\
\gamma=Z f\left(V X^{2}+Y^{2}+Z^{2}\right),
\end{array}\right.
$$

wo $f$ eine Function ist, die abnimmt, wenn ihr Argument wächst.

Nun stelle man sich in einer beliebig gestalteten Eisenmasse, die magnetisirenden Kräften ausgesetzt ist, zwei concentrische Kugelflächen, $S$ und $\boldsymbol{S}^{\boldsymbol{\prime}}$ vor: beide unendlich klein, die zweite aber gegen die erste unendlich gross. In beiden Kugeln können die magnetischen Momente, reducirt auf die Volumen-Einheit, als constant betrachtet werden; die Kräfte, die auf die Puncte der kleineren Kugel von den magnetischen Molecülen, die zwischen den beiden Kugelflächen liegen, ausgeübt werden, heben daher einander auf. Die kleinere Kugel wird demnach in ihrem magnetischen Zustande durch die Kräfte, welche von Aussen her auf die Eisenmasse wirken erhalten, so wie von den Kräften, die von den magnetischen Molecülen dieser, mit Ausschluss der Kugel $S^{\prime}$, ausgeübt werden. Alle diese Kräfte können innerbalb der Kugel $S$ als constant angesehen werden; man darf daher die Gleichungen (20) auf diese Kugel anwenden, wenn man unter $X, Y, Z$ die Componenten der Resultante des genannten Kräfte versteht. Es seien $x, y, z$ die Coordinaten eines Puncts im Innern der Kugel $S, V$ sei das Potential der äusseren Kräfte in Beziehung auf diesen Punkt, und $(X),(Y),(Z)$ seien die Kräfte, die von der magnetisirten Eisenmasse, mit Ausschluss der Kugel $S^{\prime}$, auf denselben Punct ausgeübt werden; dann erhält man:

$$
\begin{aligned}
& X=-\frac{\partial V}{\partial x}+(X) \\
& Y=-\frac{\partial V}{\partial y}+(Y) \\
& Z=-\frac{\partial V}{\partial z}+(Z)
\end{aligned}
$$


Setzt man

$$
\text { (21.) } U=\int d x^{\prime} d y^{\prime} d z^{\prime}\left(a^{\prime} \frac{\partial \frac{1}{\varepsilon}}{\partial x^{\prime}}+\beta^{\prime} \frac{\partial \frac{1}{\varepsilon}}{\partial y^{\prime}}+\gamma^{\prime} \frac{\partial \frac{1}{\varepsilon}}{\partial z}\right) \text {, }
$$

wo $x^{\prime}, y^{\prime}, z^{\prime}$ die Coordinaten eines beliebigen Puncts der Eisenmasse, $\alpha^{\prime}, \beta^{\prime}, \gamma^{\prime}$ die entsprechenden magnetischen Momente bedeuten, $\varepsilon$ die Entfernung dieses Puncts von dem Puncte $(x, y, z)$ bezeichnet, und die Integration über die ganze Eisenmasse auszudehnen ist, so ist, wie Poisson gezeigt hat:

$$
\begin{aligned}
& (X)=-\frac{\partial U}{\partial x}+\frac{4 \pi}{3} \alpha \\
& (Y)=-\frac{\partial U}{\partial y}+\frac{4 \pi}{3} \beta \\
& (Z)=-\frac{\partial U}{\partial z}+\frac{4 \pi}{3} \gamma
\end{aligned}
$$

Es wird daher:

$$
\begin{aligned}
& X=-\frac{\partial(V+U)}{\partial x}+\frac{4 \pi}{3} \alpha \\
& Y=-\frac{\partial(V+U)}{\partial y}+\frac{4 \pi}{3} \beta \\
& Z=-\frac{\partial(V+U)}{\partial z}+\frac{4 \pi}{3} \gamma,
\end{aligned}
$$

oder, wenn man $\varphi$ durch die Gleichung

definirt:

(22.)

$$
\begin{aligned}
& V+U+\varphi=0 \\
& X=\frac{\partial \varphi}{\partial x}+\frac{4 \pi}{3} \alpha \\
& Y=\frac{\partial \varphi}{\partial y}+\frac{4 \pi}{3} \beta \\
& Z=\frac{\partial \varphi}{\partial z}+\frac{4 \pi}{3} \gamma .
\end{aligned}
$$

Substituirt man diese Werthe in die Gleichungen (20), nachdem man dieselben auf die Form:

$$
\begin{aligned}
& X=\alpha f_{1}\left[V\left(\alpha^{2}+\beta^{2}+\gamma^{2}\right)\right] \\
& Y=\beta f_{1}\left[V\left(\alpha^{2}+\beta^{2}+\gamma^{2}\right)\right. \\
& Z=\gamma f_{1}\left[V\left(\alpha^{2}+\beta^{2}+\gamma^{2}\right)\right]
\end{aligned}
$$


gebracht hat, so erhält man :

$$
\begin{aligned}
& \frac{\partial \varphi}{\partial x}=\alpha\left[f_{1}\left(V\left(\alpha^{2}+\beta^{2}+\gamma^{2}\right)\right)-\frac{4 \pi}{3}\right] \\
& \frac{\partial \varphi}{\partial y}=\beta\left[f_{1}\left(V\left(\alpha^{2}+\beta^{2}+\gamma^{2}\right)\right)-\frac{4 \pi}{3}\right] \\
& \frac{\partial \varphi}{\partial z}=\gamma\left[f_{1}\left(V\left(\alpha^{2}+\beta^{2}+\gamma^{2}\right)\right)-\frac{4 \pi}{3}\right],
\end{aligned}
$$

und hierạus :

$$
\text { (23.) }\left\{\begin{array}{l}
\alpha=\frac{\partial \varphi}{\partial x} F\left[V\left(\left(\frac{\partial \varphi}{\partial x}\right)^{2}+\left(\frac{\partial \varphi}{\partial y}\right)^{2}+\left(\frac{\partial \varphi}{\partial z}\right)^{2}\right)\right] \\
\beta=\frac{\partial \varphi}{\partial y} F\left[V\left(\left(\frac{\partial \varphi}{\partial x}\right)^{2}+\left(\frac{\partial \varphi}{\partial y}\right)^{2}+\left(\frac{\partial \varphi}{\partial z}\right)^{2}\right)\right] \\
\gamma=\frac{\partial \varphi}{\partial z} F\left[V\left(\left(\frac{\partial \varphi}{\partial x}\right)^{2}+\left(\frac{\partial \varphi}{\partial y}\right)^{2}+\left(\frac{\partial \varphi}{\partial z}\right)^{2}\right)\right] .
\end{array}\right.
$$

Bezeichnet man den dem Puncte $\left(x^{\prime}, y^{\prime}, z^{\prime}\right)$ entsprechenden Werth von $\varphi$ mit $\varphi^{\prime}$, und setzt

$$
F\left[V\left(\left(\frac{\partial \varphi^{\prime}}{\partial x^{\prime}}\right)^{2}+\left(\frac{\partial \varphi^{\prime}}{\partial y^{\prime}}\right)^{2}+\left(\frac{\partial \varphi^{\prime}}{\partial z^{\prime}}\right)^{2}\right)\right]=F^{\prime},
$$

so erhält man aus (21):

$$
U=\int\left(\frac{\partial \varphi^{\prime}}{\partial x^{\prime}} \cdot \frac{\partial \frac{1}{\varepsilon}}{\partial x^{\prime}}+\frac{\partial \varphi^{\prime}}{\partial y^{\prime}} \cdot \frac{\partial \frac{1}{\varepsilon}}{\partial y^{\prime}}+\frac{\partial \varphi^{\prime}}{\partial z^{\prime}} \cdot \frac{\partial \frac{1}{\varepsilon}}{\partial z^{\prime}}\right) F^{\prime} d x^{\prime} d y^{\prime} d z^{\prime}
$$

Die Gleichungen (22 und 24) sind es, durch welche die Gleichungen (1 und 2) bei der jetzigen Annahme ersetzt werden müssen; sie werden mit denselben identisch, wenn man $F$ als constant betrachtet und $=k$ macht. Ist die Function $F$ bekannt, und hat man aus (22 und 24) $q$ bestimmt, so geben die Gleichungen (23) die magnetischen Momente im Innern des Körpers, und der Ausdruck für $U$ in (24) giebt, wenn der Punct $(x, y, z)$ ausserhalb der Eisenmasse angenommen wird, das Potential derselben in Beziehung auf diesen Punct an.

Ist der Eisenkörper ein Ellipsoïd, und ist die Kraft, die auf denselben wirkt; eine constante, so genügt man den Gleichungen (22 und 24) welches auch die Function $F$ sei, wenn man $\varphi$ einer linearen Function von $x, y, z$ gleich macht; d. h. das Ellipsoïd wird auch bei der jetzt zu Grunde gelegten Annahme gleichmässig magnetisirt. Ist

$$
V=-(a x+b y+c z)
$$

Crelle's Journial f. d. M. Bd. XLVIII. Heft 4. 
und sind die Haupt-Axen des Ellipsoïds die Coordinaten-Axen, so hat man

$$
\varphi=\frac{a}{1+k A^{0}} x+\frac{b}{1+k B^{0}} y+\frac{c}{1+k C^{0}} z
$$

zu setzen, wo $A^{0}, B^{0}, C^{0}$ dieselbe Bedingung haben wie in den Gleichungen (17) und wo $k$ aus der Gleichung

$$
F\left(V\left(\frac{a^{2}}{\left(1+k A^{0}\right)^{2}}+\frac{b^{2}}{\left(1+k B^{0}\right)^{2}}+\frac{c^{2}}{\left(1+k C^{0}\right)^{2}}\right)\right)=k
$$

zu bestimmen ist.

Weber hat in seinen electrodynamischen Maassbestimmungen S. 569 Messungen mitgetheilt, die er über das magnetische Moment eines Eisenstäbchens angestellt hat, welches einer, in der Richtung der Axe wirkenden, für alle seine Puncte constanten Kraft unterworfen war. Substituirt man mit $W$ eber für die cylindrische Form des Eisens eine ihr möglichst nahe kommende ellipsoïdische, so kann man aus diesen Messungen, und den eben angegebenen Gleichungen die Werthe der Function $\boldsymbol{F}$ für gewisse Werthe ihres Arguments berechnen. In der folgenden Tafel sind die Ergebnisse dieser Rechnung zusammengestellt; das Argument der Function $F$ ist mit $u$ bezeichnet; die mit No. überschriebenen Columnen enthalten die Nurnmern der Weber'schen Versuche, aus denen die nebenstehenden Zahlen hergeleitet sind.

\begin{tabular}{r|r|r|r|r|r}
$\mathcal{N} \varrho^{\circ}$ & $u$ & $F(u)$ & $\mathcal{N} \varrho$ & $u$ & $F(u)$ \\
\hline 1 & 301 & 23,5 & 8 & 2484 & 5,6 \\
2 & 823 & 13,5 & 9 & 1975 & 6,7 \\
3 & 1184 & 10,2 & 10 & 1583 & 8,1 \\
4 & 1512 & 8,4 & 11 & 1297 & 9,5 \\
5 & 1773 & 7,4 & 12 & 967 & 12,0 \\
6 & 2080 & 6,4 & 13 & 612 & 16,9 \\
7 & 2397 & 5,7 & 14 & 296 & 25,0
\end{tabular}

$F(u)$ ist eine reine $Z a h l ; u$ aber ist eine Kraft. Als Einheit derselben ist diejenige Kraft vorausgesetzt, die auf die Masse eines Milligramms wirkend, in einer Secunde eine Geschwindigkeit von einem Millimeter hervorbringt; es ist dies dieselbe Einheit, bei deren Zugrundelegung Gauss in seiner Abhandlung: "Intensitas vis magneticae etc." die horizontale Componente des Erdmagnetismus zu Göttingen $=1,78$. findet. 
Weber und Müller sind durch ihre Versuche auf den Schluss geführt, dass das magnetische Moment eines Eisenkörpers sich einer endlichen Grenze nähert, wenn die Intensität der magnetisirenden Kraft ins Unendliche wächst; hieraus folgt in Bezug auf die Function $F$, dass für ein unendlich grosses $u$,

$$
F(u)=\frac{K}{u}
$$

ist, wo $k$ eine Constante bedeutet. Man sieht dieses leicht, wenn man sich den Eisenkörper als ein Ellipsoïd vorstellt, welches durch eine in der Richtung einer Axe wirkende Kraft magnetisirt wird.

Ist der Eisenkörper ein unbegrenzter und unendlich dünner Cylinder, und sind überdies die Mittelpuncte der magnetisirenden Kräfte symmetrich um die Axe vertheilt, so dass, nach der Bezeichnung in (19) $V=V^{(0)}$ ist, so genügt man den Gleichungen (22 u. 24) durch die Annahrne:

$$
\varphi=-V \quad, \quad U=\mathbf{0}
$$

Dass die Gleichung (24) durch dieselbe erfüllt wird, geht aus folgender Betrachtung hervor: Wenn der Radius des Cylinders unendlich klein ist, so werden zu dem Werthe des Integrals $U$, in welchem $\varphi$ durch $-V$, oder $\varphi^{\prime}$ durch - $V^{\prime}$, ersetzt angenommen werden soll, diejenigen Theile des Cylinders, welche in endlicher Entfernung von dem Puncte $(x, y, z)$ liegen, nur etwas verschwindend Kleines beitragen. Man wird daher bei der Integration $\frac{\partial V^{\prime}}{\partial x^{\prime}}, \frac{\partial V^{\prime}}{\partial y^{\prime}}$, $\frac{\partial V^{\prime}}{\partial z^{\prime}}$, und folglich $F^{\prime}$ als constant betrachten dürfen; die zu beweisende Gleichung geht hiernach in

$$
0=\int d x^{\prime} d y^{\prime} d z^{\prime}\left(\frac{\partial V^{\prime}}{\partial x^{\prime}} \cdot \frac{\partial \frac{1}{\varepsilon}}{\partial x^{\prime}}+\frac{\partial V^{\prime}}{\partial y^{\prime}} \cdot \frac{\partial \frac{1}{\varepsilon}}{\partial y^{\prime}}+\frac{\partial V^{\prime}}{\partial z^{\prime}} \cdot \frac{\partial \frac{1}{\varepsilon}}{\partial z^{\prime}}\right)
$$

über. Die Richtigkeit hiervon folgt aber daraus, dass bei der Poisson schen Theorie- in dem hier betrachteten Falle durch die Annahme (26) den Bedingungen der Aufgabe genügt wird; wie in ( $(7)$ gezeigt worden ist.

Die magnetischen Momente $\alpha, \beta, \gamma$ in dem unendlich dünnen Cylinder haben folgende Werthe: 


$$
\text { (27.) }\left\{\begin{array}{l}
\left.\alpha=-\frac{\partial V}{\partial x} \cdot F\left(V /\left(\frac{\partial V}{\partial x}\right)^{2}+\left(\frac{\partial V}{\partial y}\right)^{2}+\left(\frac{\partial V}{\partial z}\right)^{2}\right]\right) \\
\beta=-\frac{\partial V}{\partial y} \cdot F\left(V\left[\left(\frac{\partial V}{\partial x}\right)^{2}+\left(\frac{\partial V}{\partial y}\right)^{2}+\left(\frac{\partial V}{\partial z}\right)^{2}\right]\right) \\
\gamma=-\frac{\partial V}{\partial z} \cdot F\left(V\left[\left(\frac{\partial V}{\partial x}\right)^{2}+\left(\frac{\partial V}{\partial y}\right)^{2}+\left(\frac{\partial V}{\partial z}\right)^{2}\right]\right) .
\end{array}\right.
$$

Ich bemerke noch, dass diese Ausdrücke für $\alpha, \beta, \gamma$ auch gelten, wenn die Gestalt der Eisenmasse eine ganz beliebige ist, sobald die magnetisirenden Kräfte von unendlich grosser Intensität sind; es ist dann nämlich $V$ unendlich gross, $U$ bleibt endlich in Folge der durch die Gleichung (25) ausgesprochenen Eigenschaft der Function $F$. Aus der Gleichung (22) folgt also, wenn man eine endliche Grösse gegen unendlich grosse vernachlässigt:

$$
\varphi=-V
$$

und hieraus ergeben sich unmittelbar die Gleichungen (27). Durch Benutzung von (25) kann man diese hier auf die Form bringen:

$$
\begin{gathered}
\alpha=-\frac{\frac{\partial V}{\partial x}}{V\left[\left(\frac{\partial V}{\partial x}\right)^{2}+\left(\frac{\partial V}{\partial y}\right)^{2}+\left(\frac{\partial V}{\partial z}\right)^{2}\right]} K, \beta=-\frac{\frac{\partial V}{\partial y}}{V\left[\left(\frac{\partial V}{\partial x}\right)^{2}+\left(\frac{\partial V}{\partial y}\right)^{2}+\left(\frac{\partial V}{\partial z}\right)^{2}\right]} K . \\
\gamma=-\frac{\frac{\partial V}{\partial z}}{V\left[\left(\frac{\partial V}{\partial x}\right)^{2}+\left(\frac{\partial V}{\partial y}\right)^{2}+\left(\frac{\partial V}{\partial z}\right)^{2}\right]} K .
\end{gathered}
$$

Diese Form zeigt, dass die magnetische Intensität in allen Puncten der Eisenmasse dieselbe ist, nämlich $=K$; die magnetische Axe fällt überall mit der Rịchtung der magnetisirenden Kraft zusammen.

Breslau, im Juni 1853. 